\title{
Simulation and Analysis of Cascading Failures on an NPCC Power System Test Bed
}

\author{
Wenyun Ju, Junjian Qi, Kai Sun \\ The University of Tennessee \\ Knoxville, TN, USA \\ wju1@vols.utk.edu, junjian.qi.2012@ieee.org, kaisun@utk.edu
}

\begin{abstract}
This paper develops a test bed based on the Northeastern Power Coordinating Council (NPCC) 48-machine, 140-bus power system model for simulating cascading failures. Then, the paper uses the test bed to demonstrate the interaction model recently proposed by [1] for analyzing cascading failures, identifying key linkages between component failures, and predict propagation of failures. For the NPCC test bed, a new efficient method is proposed to determine line flow limits as critical parameters in cascading failures simulation. Then, those limits are utilized in AC-OPA to generate a database of cascades. Finally, the interaction model is derived based on the database to extract key knowledge on cascades, which can be used for online analysis and simulation of cascading failures.
\end{abstract}

Index Terms-Blackout; cascading failures; interaction model; AC-OPA; DC-OPA; Northeastern Power Coordinating Council (NPCC) Power System; line flow limit.

\section{INTRODUCTION}

Cascading failures propagating in power grids may lead to catastrophic blackouts, e.g. the blackouts in North America on 14th August 2003 [2], in Brazil on 10th November 2009 [3], and in India on 30th and 31st July 2012 [4]. To analyze cascading failures and evaluate their influences on power grids, several models have been proposed, such as the Manchester model [5], CASCADE model [6], hidden failure model [7]-[9], branching process model [10], [11], and OPA ${ }^{1}$ model [12]-[16]. Manchester model considers overloads, relay malfunction, and transient instability, corresponding to a relatively detailed simulation. The hidden failure model emphasizes the influence of relay hidden failures. CASCADE and branching process model are high-level probabilistic models that describe the cascading process but do not model power system physics. Based on Self-Organized Criticality theory and optimal power flow calculation, OPA model simplified steady-state behaviors of a real power grid in two time scales: slow dynamics, i.e. the growth of load, generation and line capacities over years to meet reliability requirements, and fast dynamics representing cascading line overloads and outages under specific cascading events. A typical OPA model is based on DC power flow, which is called DC-OPA in this paper, while an AC-OPA model [15] is more accurate thanks to its AC optimal power flow engine considering voltage variations and reactive power.

This work was supported by the University of Tennessee in Knoxville and the CURENT Engineering Research Center.

IOPA stands for Oak Ridge National Laboratory, Power Systems Engineering Research Center at University of Wisconsin, and University of Alaska to indicate the institutions involved in the collaborating effort.
The "influence model" which is a tree network is proposed to quantify the influences between the components of network [17]. Similar to this general idea, [18] quantifies the interactions between transmission lines by line interaction graph in order to analyze cascading failures. For fast analysis and simulation of cascading failures, paper [1] proposes a power system's interaction model that is constructed based on a database of cascades from either historical events or simulations. That interaction model extracts key information on cascading failures of the power system, quantifying how interactions between component failures influence the risk of cascading failures and capturing general propagation patterns of cascades. Thus, scenarios of cascading failures can be simulated and analyzed quickly and effectively in a time-intensive environment only using that interaction model for prediction of their propagations and mitigation actions without need to conduct time-consuming simulation of the original power system model. Thus, the interaction model if available for a power system can readily fit into real-time operation for operators' situational awareness and decision support.

This paper demonstrates the application of the interaction model to a realistic power system model. Major contributions of this paper include: first, developing a cascading failures simulation test bed for the Northeastern Power Coordinating Council (NPCC) power system based on its 48-machine, 140bus reduced model, whose line flow limits as critical parameters in cascading failures simulation are determined by a new efficient method based on DC-OPA; second, demonstrating the interaction model built from a huge number of original cascades simulated by applying AC-OPA on that test bed, and further comparing the cascades simulated by only using the interaction model with those original cascades in terms of key linkages between component failures and the spread patterns of cascades. The paper also intends to give a detailed procedure to follow on how to utilize the interaction model for analysis and simulation of cascading failures. The rest of this paper is organized as follows. Section II introduces the interaction model briefly. Section III proposes a new method to obtain the line flow limits for cascading failures simulation and illustrates it on the NPCC test bed. Section IV demonstrates the interaction model built from a database of cascades simulated by an ACOPA based fast dynamic process on the test bed. Section V draws some conclusions. 


\section{INTERACTION MODEL}

This section briefly introduces concepts and indices about the interaction model that will be used later in section IV. Details on the model can be found in [1].

\section{A. Original Cascades and Simulated Cascades}

In this paper, "original cascades" are the cascading failure sequences from utilities or generated by detailed cascading failure models while "simulated cascades" are the cascading failures sequences produced directly by the interaction model that is built from "original cascades".

\section{B. Interaction Matrix and Interaction Network}

The interaction matrix $B$ determines how components (e.g. lines) interact with each other based on the original cascades. The nonzero element $b_{i j}$ of $B$ is the empirical probability that the failure of component $i$ triggers the failure of component $j$. The interaction matrix can be represented by the interaction network or graph $\varsigma(C, L)$, for which each link corresponds to one nonzero element in $B$ and represents that a failure of the source vertex component causes the failure of the destination vertex component with nonzero probability. The vertex set $\mathrm{C}$ represents all components of the system.

\section{Key Links and Components Identification}

In [1] the link weight $I_{l}$ is defined to indicate the contribution of a link $l$ to the propagation of cascading failures. The link weight is actually the expected value of the number of failures that are propagated through the link. Then, the set of key links $L^{k e y}$ can be obtained by

$$
L^{k e y}=\left\{l \mid I_{l} \geq \varepsilon_{l} I_{l}^{\max }\right\}
$$

where $\varepsilon_{l}>0$ is a constant to identify some key links with large link weight, $I_{l}^{\max }$ is the largest link weight of all links.

The vertex out-strength of the interaction network indicating how much a component $i$ influences another is

$$
S_{i}^{\text {out }}=\sum_{l \in L^{\text {uut }}(i)} I_{l}
$$

where $L^{\text {out }}(i)$ is the set of links starting from vertex $i$. The set of key components $C^{k e y}$ which contribute mostly to the propagation of cascading failures can be obtained by

$$
C^{\text {key }}=\left\{i \mid S_{i}^{\text {out }} \geq \varepsilon_{s} S_{i}^{\text {out } \text { max }}\right\}
$$

where $\varepsilon_{s}>0$ is a constant to identify key components with large out-strengths, and $S_{i}^{\text {out,max }}$ is the largest one.

\section{Interaction Model}

As in [1], an interaction model can be used to efficiently simulate cascades based on the initial tripping probability of each component and the interaction matrix $B$. All components are assumed to be initially operating and each component fails with a small probability. The component failures then cause other component failures independently according to the empirical probability in interaction matrix $B$.

\section{E. Mitigation Measures of Cascading Failures}

Since key links contribute much to propagation of cascading failures, which may be mitigated by weakening the corresponding element in interaction matrix $B$. Thus, mitigation measures may be suggested, e.g. blocking relays on the destination vertices and even controlled islanding [19] to break some key lines and isolate cascades. A random mitigation measure means weakening elements in $B$ randomly while an intentional mitigation measure chooses key links in matrix $B$ to weaken. Section IV will investigate effectiveness of mitigation measures in reducing risks of cascading failures.

\section{Determining The Line Flow Limits}

In order to apply the interaction model to the NPCC test bed, we first generate original cascades by using the fast dynamic process of AC-OPA model for which reasonable line flow limits as important parameters are needed. In [11], the line flow limits are determined by running the fast dynamics of OPA and the slow dynamics of OPA on a base load level that together emulate a long-term power system planning process to selectively upgrade lines in response to their involvements in daily failures starting from initial guesses of the limits. Then the line flow limits at an expected load level (either the base load level or a future level) are determined by

$$
\text { Limit }_{i}^{D C}=\text { Limit }_{i}^{k} / \lambda^{m}
$$

where Limit $_{i}{ }^{D C}$ represents the line flow limit of component $i$ at the expected load level, Limit $_{i}^{k}$ is the line flow limit of component $i$ on $k$-th simulation day, $\lambda>1$ is a constant, representing mean load daily growth factor, and $m \leq k$. The line flow limits to be determined are for the base load level if $m=k$, or a targeting future load level if $m<k$. In this paper, satisfaction to the $\mathrm{N}-1$ criterion on each simulation day is not required. However, the OPA algorithm can easily be modified to consider the N-1 criterion. For example, [13] extended the OPA simulation to address the $\mathrm{N}-1$ criterion.

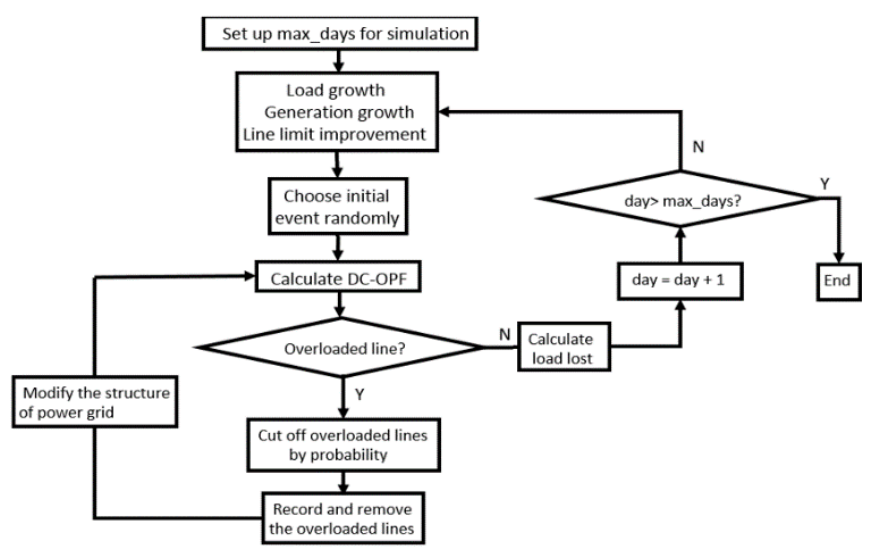

Fig. 1. Flow chart of the DC-OPA method for line flow limits

Fig.1 describes an OPA-based method for obtaining the line flow limits. The advantage of using DC-OPA is fast and easy to solve. However, such a DC-OPA method when determining line flow limits does not consider the influence of reactive power. In contrast, the fast dynamic process of AC- OPA to produce a database of original cascades does consider reactive power and voltage variations. That cause some limits from DC- 
OPA to be violated at high probabilities for lines carrying heavy reactive power flows, e.g. those supporting load center areas.

Here we have to mention that for a power system, if each bus has a high power factor ( $>0.76$ for the IEEE 118 -bus system studied in [1] and [11]), the impact of reactive power on violations of the line flow limits from the DC-OPA method during the fast dynamic process of AC-OPA is minor. In that case, the limits from the DC-OPA method can directly be used. However, for the NPCC test bed, there are a few load buses with very low power factors, e.g. buses 3, 92, 95 and 114 since in those areas, there are a few lines carrying heavy reactive power flows to support voltage. The limits from the DC-OPA method will be violated in the fast dynamic process of AC-OPA. Therefore, for some power system like the NPCC test bed, we need to revise the line flow limits obtained by DC-OPA based method using (5) to give considerations to the impact from reactive power, where $\operatorname{Limit}_{i}^{A C}$ and $\operatorname{Limit}_{i}^{D C}$ are respectively a corrected line flow limit and the limit obtained from the original DC-OPA method, and $Q_{i}$ is the planned reactive power flow of component $i$ at the expected load level, which may approximately be estimated by, e.g., the reactive power flow at the base load level multiplied by $\lambda^{k-m}$ if there is no better knowledge on it.

$$
\operatorname{Limit}_{i}^{A C}=\sqrt{\left(\text { Limit }_{i}^{D C}\right)^{2}+Q_{i}^{2}}
$$

Alternatively, an AC-OPA method may substitute AC OPF for DC OPF in the procedure of Fig. 1 for more accurate limits addressing reactive power. However, that will cause greatly increased computation burdens but the limits obtained will have similar statistical properties with the limits obtained by the aforementioned modified DC-OPA method.

\section{SimUlation RESUlts}

\section{A. Determining Line Flow Limits for the NPCC Test Bed}

The original NPCC 140-bus, 48-machine, 233-branch model comes from the Power System Toolbox [20] and represents the backbone transmission of the northeast region of the Eastern Interconnection, which was involved in the 2003 blackout event. The base load level of the model is $28 \mathrm{GW}$. We use constant P/Q load models. The first task is to obtain a set of reasonable line flow limits for that base load level such that a test bed is developed for cascading failures simulation.

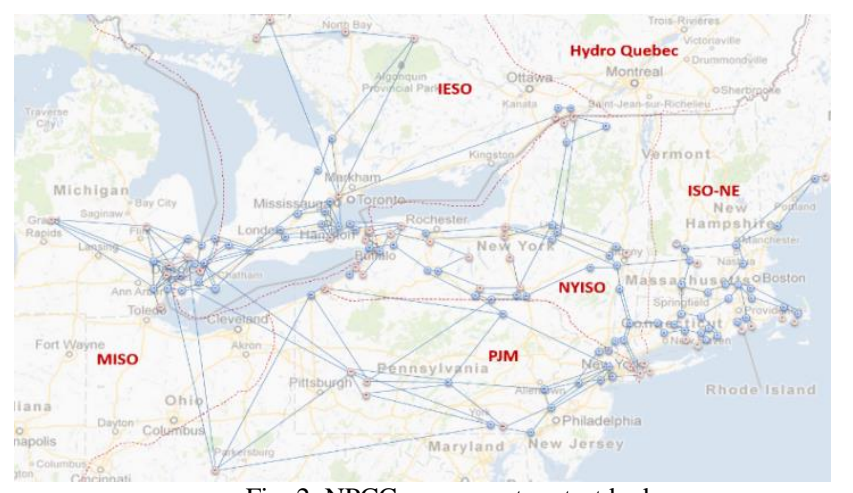

Fig. 2. NPCC power system test bed

The $2^{\text {nd }}$ column of Table I gives main parameters of the original DC-OPA method. Each initial line flow limit takes double of the base line flow. After a set of line flow limits $\operatorname{Limit}_{i}^{D C}$ is obtained. The final line flow limits Limit $_{i}^{A C}$ are calculated from (5). Then, the test bed is simulated by an ACOPA based fast dynamic process to generate the original cascades, whose parameters are given in the $3^{\text {rd }}$ column.

TABLE I. PARAMETERS OF DC-OPA

\begin{tabular}{|c|c|c|c|}
\hline Parameter & DC-OPA & AC-OPA & Remark \\
\hline No. of days & 64000 & 10000 & Simulation days \\
\hline$\lambda$ & 1.0005 & - & Daily load growth factor \\
\hline$\mu$ & 1.005 & - & Line improvement factor \\
\hline$\gamma$ & 1.67 & 1.67 & Load variability \\
\hline $\mathrm{p}_{0}$ & 0.0001 & 0.0001 & Probability of initial line outage \\
\hline
\end{tabular}
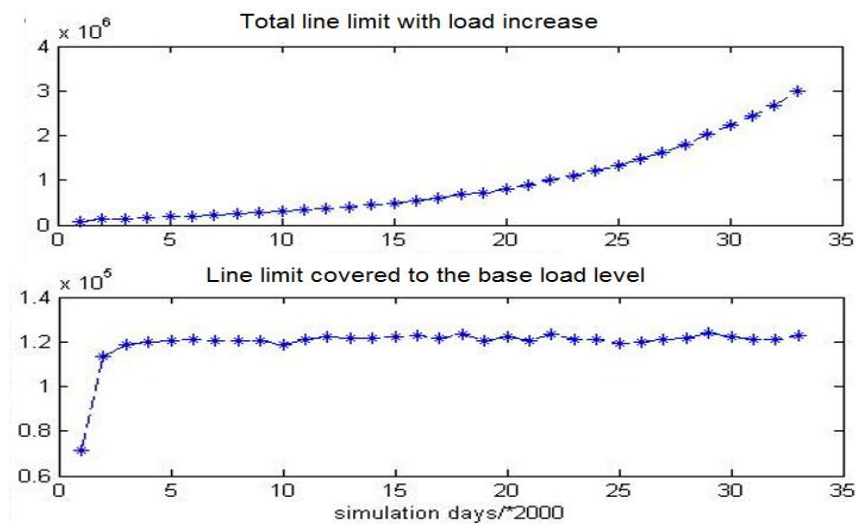

Fig. 3. Simulation results of total line flow limit before and after conversion to the basic load level

Fig. 3 gives the total line flow limit from the original DCOPA method. From it, a conclusion is that the total line flow limit is stable after the number of simulation days reaches 10000 when two strengths, the load growth to trigger cascades and the line upgrading to reduce the risk of cascades, reach their balance, i.e. the equilibrium of the slow dynamic process. We compared the average variation of each line limit between two stages, i.e. the 10000 -th day and the 64000-th day. The variation is as small as $6.9 \%$ so that we may ignore the fluctuation of the total line limit after the 10000-th day and adopt the set of line flow limits on that day as the initial limits.

TABLE II. LINE FLOW LIMITS FOR ORIGINAL DC-OPA METHOD

\begin{tabular}{|c|c|c|c|}
\hline Line No. & $\begin{array}{c}\text { MW and MVar flows } \\
\text { at base load }\end{array}$ & $\begin{array}{c}\text { Line flow limit by } \\
\text { DC-OPA }\end{array}$ & Updated limit \\
\hline 4 & $3.99 \mathrm{MW}, 43.83 \mathrm{Mvar}$ & $24.93 \mathrm{MVA}$ & $50.42 \mathrm{MVA}$ \\
\hline 5 & $5.07 \mathrm{MW}, 45.91 \mathrm{Mvar}$ & $24.17 \mathrm{MVA}$ & $51.88 \mathrm{MVA}$ \\
\hline 223 & $51.44 \mathrm{MW}, 163.09 \mathrm{Mvar}$ & $126.09 \mathrm{MVA}$ & $206.15 \mathrm{MVA}$ \\
\hline
\end{tabular}

Reactive powers of lines 4, 5 and 223 are found larger than the line flow limits from the original DC-OPA method. Table II gives those limits, their updated values by using (5), and the real and reactive power flows of those lines at the base load level for purpose. The updated line flow limits will be used in simulation to create original cascades.

\section{B. Building the Interaction Model}

Following the algorithm of the interaction model, key links and components that play important roles in the propagation of 
cascading failures are identified. Those identified key links are actually line pairs in the NPCC test bed, and their weights $I_{l}$ for the original cascades are listed in Table III.

TABLE III. KEY LINKS OF NPCC TEST BED

\begin{tabular}{|c|c|c|}
\hline$i-j$ & Line pairs & $I_{l}$ \\
\hline $199 \rightarrow 198$ & $(126,125) \rightarrow(126,124)$ & 4156.7 \\
\hline $38 \rightarrow 6$ & $(33,32) \rightarrow(4,1)$ & 3005.5 \\
\hline $112 \rightarrow 105$ & $(81,78) \rightarrow(75,76)$ & 2041.7 \\
\hline $19 \rightarrow 24$ & $(13,12) \rightarrow(15,14)$ & 1549.7 \\
\hline $38 \rightarrow 8$ & $(33,32) \rightarrow(5,31)$ & 1377.1 \\
\hline $6 \rightarrow 36$ & $(4,1) \rightarrow(31,30)$ & 1330.9 \\
\hline $21 \rightarrow 6$ & $(14,13) \rightarrow(4,1)$ & 1278.2 \\
\hline $198 \rightarrow 201$ & $(126,124) \rightarrow(127,126)$ & 1277.7 \\
\hline $198 \rightarrow 204$ & $(126,124) \rightarrow(128,126)$ & 1254.8 \\
\hline $198 \rightarrow 225$ & $(126,124) \rightarrow(138,126)$ & 1253.2 \\
\hline $112 \rightarrow 197$ & $(81,78) \rightarrow(125,124)$ & 1186.6 \\
\hline $38 \rightarrow 42$ & $(33,32) \rightarrow(35,34)$ & 928.6 \\
\hline $38 \rightarrow 40$ & $(33,32) \rightarrow(34,33)$ & 893.5 \\
\hline $18 \rightarrow 24$ & $(12,7) \rightarrow(15,14)$ & 832.6 \\
\hline $24 \rightarrow 29$ & $(15,14) \rightarrow(18,17)$ & 816.4 \\
\hline $19 \rightarrow 21$ & $(13,12) \rightarrow(14,13)$ & 784.0 \\
\hline $105 \rightarrow 215$ & $(75,76) \rightarrow(134,132)$ & 780.3 \\
\hline $19 \rightarrow 23$ & $(13,12) \rightarrow(15,7)$ & 749.6 \\
\hline $38 \rightarrow 7$ & $(33,32) \rightarrow(5,4)$ & 735.1 \\
\hline $32 \rightarrow 31$ & $(20,19) \rightarrow(20,17)$ & 721.9 \\
\hline $114 \rightarrow 166$ & $(83,112) \rightarrow(113,112)$ & 672.9 \\
\hline $197 \rightarrow 198$ & $(125,124) \rightarrow(126,124)$ & 669.1 \\
\hline $162 \rightarrow 163$ & $(111,108) \rightarrow(111,109)$ & 650.4 \\
\hline $6 \rightarrow 35$ & $(4,1) \rightarrow(30,29)$ & 643.7 \\
\hline $197 \rightarrow 203$ & $(125,124) \rightarrow(128,125)$ & 631.0 \\
\hline
\end{tabular}

TABLE IV. KEY COMPONENTS OF NPCC TEST BED

\begin{tabular}{|c|c|c|}
\hline Key component & Line & $S_{i}^{\text {out }}$ \\
\hline 38 & $(33,32)$ & 8822.2 \\
\hline 6 & $(4,1)$ & 7339.7 \\
\hline 199 & $(126,125)$ & 5914.4 \\
\hline 112 & $(81,78)$ & 5586.7 \\
\hline 105 & $(75,76)$ & 4473.7 \\
\hline 198 & $(126,124)$ & 4097.9 \\
\hline 19 & $(13,12)$ & 3664.8 \\
\hline 24 & $(15,14)$ & 3302.1 \\
\hline 166 & $(113,112)$ & 2974.0 \\
\hline 21 & $(14,13)$ & 2610.3 \\
\hline 8 & $(5,31)$ & 1994.5 \\
\hline 18 & $(12,7)$ & 1892.9 \\
\hline 197 & $(125,124)$ & 1583.9 \\
\hline 215 & $(134,132)$ & 1580.8 \\
\hline 35 & $(30,29)$ & 1470.1 \\
\hline
\end{tabular}

Complementary cumulative probability distribution of link weight of all links is displayed in Fig.4. Both $\varepsilon_{l}$ and $\varepsilon_{s}$ take 0.15 . Key links only take $0.7 \%$ of all links of the system but the sum of their weights cover $38.5 \%$ of the total weights of all links. These very few links contribute quite a lot to the propagation of cascading failures and together capture highly concentrated key information on cascading failures of the NPCC test bed. The identified key components are listed in
Table IV. Failures of these components are the most involved ones in cascades. Operators should pay more attentions to those components. The number of key components is 15 , i.e. $6.43 \%$ of all components for the original cascades. However, the sum of the out-strengths of key components is $68.8 \%$ of that for all involved components.

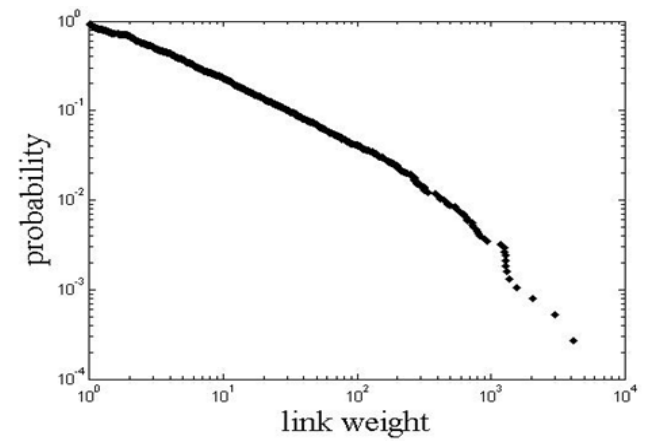

Fig. 4. Complementary cumulative probability distribution of link weight

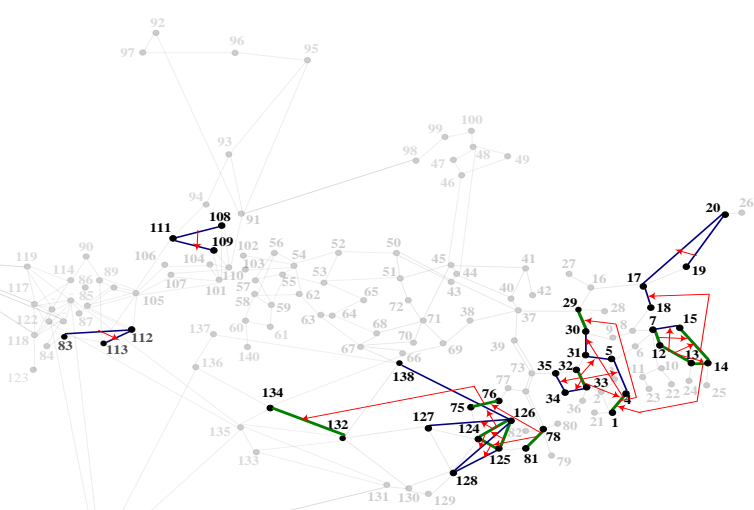

Fig. 5. Key links (red arrows), key components (green lines), and other involved components (blue lines) with the rest of the system (faded)

Fig. 5 highlights the key links (red arrows), key components (green lines) and other involved components (blue lines), which together determine the areas that are most vulnerable to cascading failures. Actually, those areas match very well the geographical locations of load center areas in the actual NPCC system such as the Connecticut load center and New York City load center. From those identified links and components, propagation paths of cascading failures are indicated, e.g. the path $(75,76) \rightarrow(134,132),(13,14) \rightarrow(4,1)$ and $(15,14) \rightarrow$ $(18,17)$. Two observations from Fig. 5 on the propagation paths are: first, the source and destination vertices of most key links, e.g. at an earlier stage of a propagation path, are lines geographically close to each other, which indicates that cascading failures often initiate from local problems; second, however, at the late stage of a path, some key links may have source and destination vertices far away from each other, which means that, at a later stage, cascading failures develop to a wider-area or even system-wide problem. The second observation indicates that the power system exhibits more nonlinear "butterfly effect" behaviors in a late stage of cascading failures, which are captured by the AC-OPA based fast dynamic process for creating the original cascades and consequently can be captured by the interaction model. This 
property is similar to the discussion in [1]. Those observations suggest that mitigation actions should be taken before cascading failures develop to a system-wide problem.

\section{Comparison between Original and Simulated Cascades}

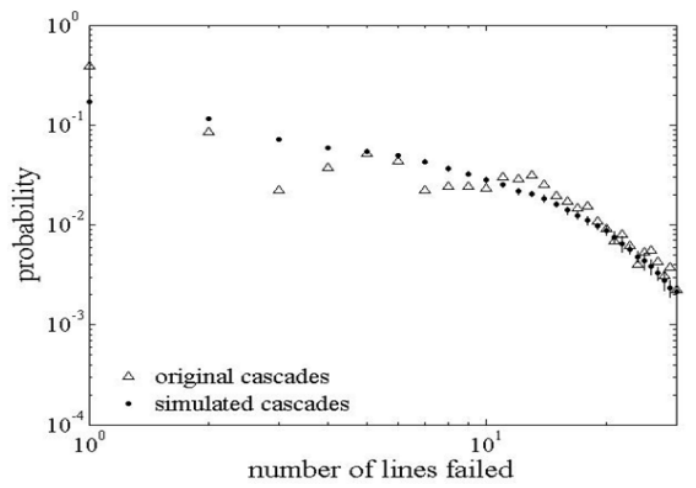

Fig. 6. Probability distributions of the total number of line failed for original and simulated cascades

The probability distributions of the total number of line outages respectively for original cascades and simulated cascades are plot in Fig. 6. As in [1], we simulate 20 times and obtain the average probability distribution and the standard deviations (vertical axis) for the simulated cascades. It is found that those two distributions basically match especially for bigger cascades (with >10 lines failed). The standard deviations for the simulated cascades are small. It indicates that the interaction model authentically captures statistical properties of the original cascades.

\section{Mitigation Measures of Cascading Failures}

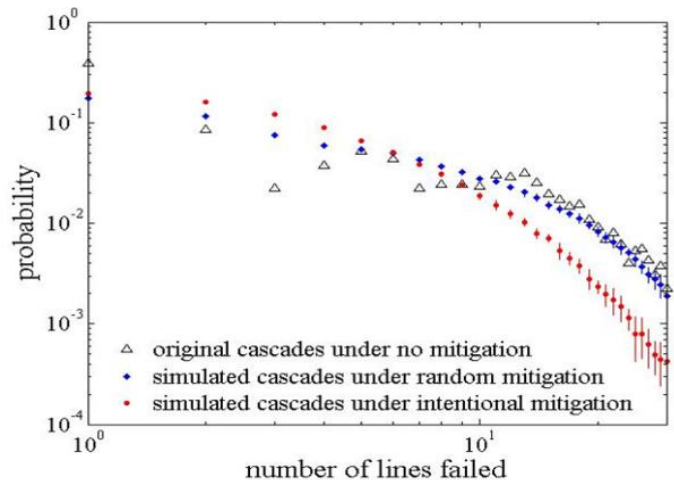

Fig. 7. Probability distributions of the total number of line failed for original and simulated cascades under different mitigation measures

Intentional mitigation measures that block some relays suggested from the interaction model are tested and compared with random relay blocking measures. From Fig. 7, the risk of large-scale cascades is greatly reduced with the intentional measures while that for the random measures is not obvious, which suggests the identified key links indeed play important roles in the propagation of cascading failures.

\section{CONCLUSION}

This paper has demonstrated the interaction model on the NPCC test bed to identify the key links and components that play important roles to the propagation of cascading failures. A new fast method for determining line flow limits has been proposed and applied to the NPCC test bed. Compared to other cascading models previously developed, the interaction model, if built offline from historical events or simulations, can be utilized in the real-time operation environment. Grid operators may use it to narrow down the range of components for realtime monitoring when the grid becomes more vulnerable to cascading failures. If cascading failures occur, the model will help operators predict paths of propagation and select effective mitigation measures by weakening the key links in the model.

\section{REFERENCES}

[1] J. Qi, K. Sun, S. Mei, "An interaction model for simulation and mitigation of cascading failures," IEEE Trans. Power Systems, in press, DOI: 10.1109/TPWRS.2014.2337284.

[2] U.S.-Canada Power System Outage Task Force. Final report on the August 14, 2003 blackout in the United States and Canada: Causes and Recommendations. April, 2004.

[3] "Dam Failure Triggers Huge Blackout in Brazil," CNN, 2009.

[4] L. Lai, H. Zhang, C. Lai, et al, "Investigation on July 2012 Indian blackout,", Proceedings of the 2013 International Conference on Machine Learning and Cybernetics, Tianjin, 14-17 July, 2013.

[5] M. A. Rios, D. S. Kirschen, D. Jawayeera, et al, "Value of security: modeling time-dependent phenomena and weather conditions," IEEE Trans. Power Systems, vol. 17, pp. 543-548, Aug. 2002.

[6] I. Dobson, et al, "A probabilistic loading dependent model of cascading failure and possible implications for blackouts," 36th Annu. Hawaii Int. Conf. System Sciences, Jan. 2003, pp. 65-74.

[7] J. Chen, J. S. Thorp, I. Dobson. "Cascading dynamics and mitigation assessment in power system disturbances via a hidden failure model". Int. J. Elect. Power Energy Syst., vol. 27, no. 4, pp. 318-326, 2005.

[8] A. G. Phadeke, J. S. Thorp, "Expose hidden failures to prevent cascading outages," IEEE Comput. Appl. Power, vol. 9, no. 3, pp. 20-23, 1996.

[9] S. Tamaronglak, "Analysis of power system disturbances due to relay hidden failures," Ph.D. dissertation, Virginia Poly. and State Univ., Blacksburg, VA, 1994.

[10] I. Dobson, et al, "Testing branching process estimators of cascading failure with data from a simulation of transmission line outages," Risk Analysis, vol. 30, pp. 650-662, Apr. 2010

[11] J. Qi, I. Dobson, S. Mei, "Towards estimating the statistics of simulated cascades of outages with branching processes," IEEE Trans. Power Systems, vol. 28, pp. 3410-3419, Aug. 2013.

[12] I. Dobson, B. Carreras, V. Lynch, D. Newman, "An initial model of complex dynamics in electric power system blackouts," 34th Аnnu. Hawaii Int. Conf. System Sciences, 2001, pp. 710-718.

[13] H. Ren, I. Dobson, B. A. Carreras, "Long-term effect of the n-1 criterion on cascading line outages in an evolving power transmission grid," IEEE Trans. Power Systems, vol. 23, pp. 1217-1225, Aug. 2008.

[14] S. Mei, et al, "An improved OPA model and blackout risk assessment," IEEE Trans. Power Systems, vol. 24, pp. 814-823, May. 2009.

[15] S. Mei, et al, "A study of self-organized criticality of power system under cascading failures based on AC-OPA with voltage stability margin," IEEE Trans. Power Syst., vol. 23, pp. 1719-1726, Nov. 2008.

[16] J. Qi, S. Mei, F. Liu, "Blackout model considering slow process," IEEE Trans. Power Systems, vol. 28, pp. 3274-3282, Aug. 2013.

[17] S. Roy, et al, "Network models: growth, dynamics, and failure," 34th Annu. Hawaii Int. Conf. System Sciences, Jan. 2001.

[18] P. D. Hines, et al, “'Dual Graph' and 'Random Chemistry' methods for cascading failure analysis," 46th Hawaii Intl. Conference on System Sciences, Jan. 2013.

[19] K. Sun, K. Hur, P. Zhang, "A New Unified Scheme for Controlled Power System Separation Using Synchronized Phasor Measurements", IEEE Trans. Power Systems, vol. 26, pp. 1544-1554, Aug. 2011

[20] J. Chow, G. Rogers, "User Manual for Power System Toolbox, Version 3.0," 1991-2008. 